ORIENTAL JOURNAL OF
ISSN: 0974-6471
June 2017,
COMPUTER SCIENCE \& TECHNOLOGY

\title{
Trends of Manufacturing Systems with Distributed Computing
}

\author{
ABEDALHAKEEM T. E. ISSA \\ Computer Science Department DCC Shaqrs University, Kingdom Saudi Arabia \\ Corresponding author E-mail: dr_abedalhakeemi@yahoo.com
}

http://dx.doi.org/10.13005/ojcst/10.02.02

(Received: June 01, 2017; Accepted: June 17, 2017)

\begin{abstract}
The industry developed dramatically in the second half of the 20th century, And with it developed and manufacturing systems ranging from manual to fully computerized systems employing information and communication technology (ICT). This fact has made the manufacturing systems to be totally dependent on ICT and therefore these systems have to keep pace with the advancement in ICT. Distributed computing has totally changed the computing paradigm in recent times resulting in rapid employment of these technologies in the manufacturing sector. An important variable in the equation determining the trend of manufacturing technologies is the purchaser choice and preference which has become active recently. To address these heterogeneous user demands, the Autonomous Decentralized System (ADS) concept was introduced five decades ago. The ADS has been a significant development incorporated in modern manufacturing systems and have been standardised as the de-facto standard for factory automation. These systems hold the assure for on-line system maintenance, timeliness and assurance, ensuring greater productivity and cost benefit emerging as the system of choice in automated manufacturing systems. This paper reviews the ADS, its application to a manufacturing system, assesses the state of the art and discusses the future trends.
\end{abstract}

Keywords: Autonomous decentralized systems, manufacturing systems, distributed computing.

\section{INTRODUCTION}

Manufacturing systems have been progressing through an evolutionary process in unison with advances in computing technology $y^{1,2}$. However, the recent advancement in Information and Communication Technology (ICT) has given new impetus to the design of manufacturing systems. There is an increasing demand for complex distributed systems capable of handling large-scale factory automation. Moreover, the dynamics of economic global recession has caused a shift in the manufacturing doctrine from mass produced items to various production. The market forces are driven, 
in most part by changing consumer behaviour. It is therefore imperative for a new production system to be flexible enough to address heterogeneous user demands and trends as its core strength.

Such a production system therefore, should have the capability to continue its operation while new components are either added or removed from the main system in response to changing requirements for production. Also it should be possible to analyze and debug faults while the system is operational so that the system down-time is minimized.

Autonomous Decentralized System concept was introduced in Japan to address the design issues facing the manufacturing systems in the wake of advances in $\mathrm{ICT}^{4}$. ADS has attracted a lot of research effort around the world since its proposal. The first symposium on ADS technologies was held in 1993, second in 1995, third in 1997 followed by the most recent in 2003. All these symposiums are referred to as ISADS followed by the year in which held. The ADS architecture was eventually approved as a de-facto standard for factory automation by international consortium of factory automation ${ }^{3}$.

In this paper ADS technology will be reviewed. The basic concept of ADS will be described in the next section. Section 3 will

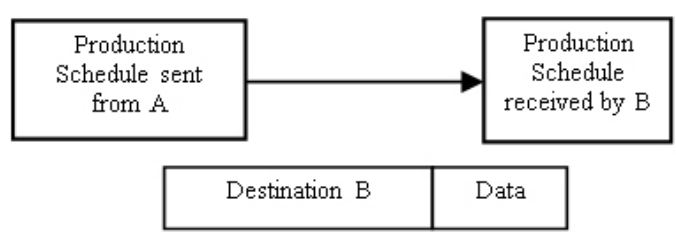

The Conventional Communication Scheme

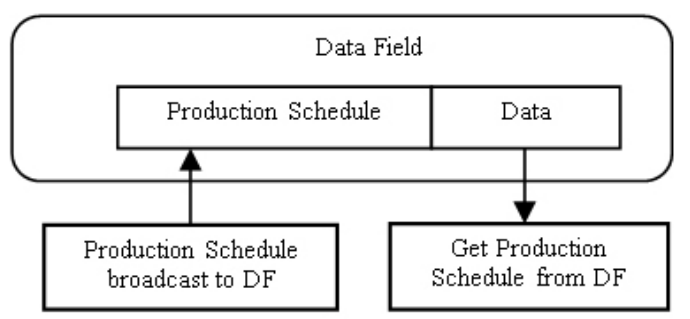

The ADS message scheme employing CC Communication

Fig. 1: Comparison of conventional and content code communication schemes consider the mode of communications used by the ADS among various modules in the architecture. Section 4 will describe the general requirements of manufacturing systems. Section 5 will outline the layout of a typical ADS whereas section 6 will look at the current state of the art of this technology and its future prospects. The paper will be concluded in section 7 .

\section{The Decentralized System}

The Autonomous Decentralized System (ADS) was proposed around four decades ago in $1984^{4}$. The concept was derived from an analogy to living organisms. Every living thing is composed of cells. Each cell is independent of other cells in the body and is totally self sufficient for the information for its living and reproduction.

None of these cells is involved in any master-slave relationship. The cells are therefore totally autonomous in their survival and function. Similarly the organisms in the living thing are performing their own function in unison with other organs in the body. The functions of the organs in the body can be characterised to be heterogeneous compared to the homogenous functions of cells. Any communication that takes place between cells and the organs does not violate their basic autonomy. In case an organ malfunctions, its function may be moved to other organs. Thus the living body carries out its function of living and growth through its cellular structure while ensuring assurance, agility, mobility and autonomy.

The ADS was proposed with two underlying assumptions with respect to the information system, i.e, the computing system will invariably have faulty parts and that it will undergo the transition of various stages of its operation including maintenance and future expansion.

In case of a homogeneous system, following are the important characteristics of the ADS

1. Defect acceptance: The partial failure of the system does not hinder the system operation in any way. The normal designed operation of the system is possible to be carried out with the added advantage that the fault will not propagate to the rest of the system.

2. Direct system growth:The system operation 
is not affected in any way when additional subsystems are added or removed.

3. Maintenance and testing: Usual maintenance and testing operations are possible to be carried out on the system without with out stopping system operation.

\section{An ADS has to satisfy the following properties if} it is employed in a heterogeneous system:

1. System flexibility: The changing environment should be matched to the dynamics of the system flexibility.

2. Mobility: The functional behaviour of the system should incorporate mobility whereby it may be able to migrate to other systems in response to changing conditions around the system.

3. Assurance: Under evolving conditions, the system should be able to collaborate with other systems in the event that it is unable to perform that function alone.

These properties of the ADS are analogous to the cells and organs in a living body. The ADS has to satisfy the characteristics of autonomous control and autonomous coordination with respect to its analogy to living organisms. Coordination and control function is the key in the assurance of online property. The addition, deletion or failure of any subsystems in the main ADS does not jeopardize the operation of the main system. The subsystems can coordinate with each other to perform their functions being totally autonomous at the same time. Each subsystem is also autonomous and does not engage in any master-slave relationship with any other subsystem. Thus all the subsystems

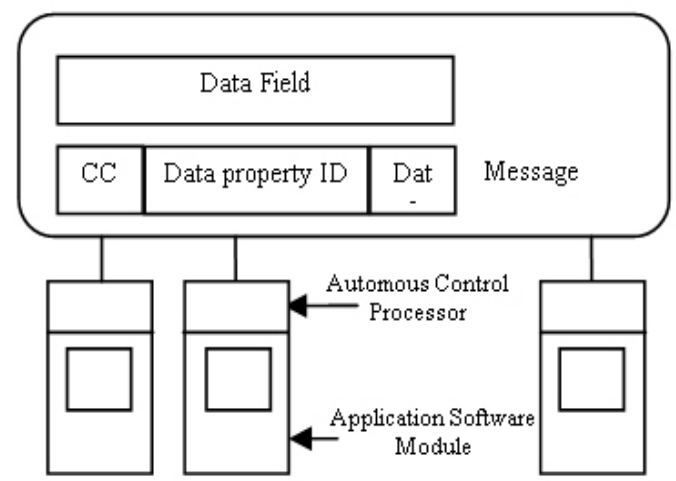

Fig. 2: The Data field architecture are equal in function. The management of each subsystem to carryout its function is local and any coordination with other subsystems is based on local information only. Therefore the condition of locality is met. Moreover the structure of each subsystem is uniform and self contained. This property makes the subsystem totally autonomous. It has then the autonomy to mange itself and carry out the necessary coordination with other subsystems. Thus the condition of uniformity is met.

\section{Types of communication with the Systems}

The deviation of ADS from conventional systems warrants a totally different approach for communication within the main system. The conservative communication methods cannot be used in ADS.

\section{Communication in Homogeneous Systems}

The ADS employs a unique method of communication referred to as content code communication. Two important components of this communication technique are the data field (DF) and the message. There is no central authority in the system. The subsystems are managed by their own processors called autonomous control processors (ACP). The subsystems communicate through a common data filed whereby the message is broadcast to the DF with the appropriate content code specifying its destination. The message is received by the intended subsystem through the DF and rejected by other subsystems. The content codes of the messages are pre-registered in the ACP of each subsystem. The comparative message passing scheme is shown in Fig. 1 for both conventional and ADS messages.

The broadcast message is deleted by the originator after it is sent to the DF. The application software module installed in a subsystem starts the execution of its function only after all the required user data is received. Thus the subsystems in ADS are loosely attached modules of a major system operating autonomously and executing their individual function. The DF architecture is depicted in Figure 2. 
In case of a heterogeneous system employing ADS technology, various application systems are integrated together through gateways ${ }^{5}$. In such a scheme, every application system is connected to its own DF to retain its own characteristics. They retain their designed functions but cooperate with other subsystems. A heterogeneous system connecting its application modules through a gateway is shown in Fig. 3. Here, the data from various DFs belonging

to various subsystems can exchange data through the gateway node. The gateway node only relays the required data to other DFs since the contents of data are known a priori to the gateway node. For example the details of sales in a sales department DF can be relayed to the production department DF on required basis.

\section{Manufacturing System structure}

Conventional manufacturing systems have a hierarchical structure and therefore they lack the much needed flexibility. The very nature of such systems is complex and it makes any modification in the system an demanding task for the engineers. The hardware and software structures of these systems tend to be complex since the two important entities of the system, i.e, information and control subsystems are prone to generate more communication traffic as the manufacturing systems are getting more information- intensive. In case of any addition/deletion in the main system, the engineers have to comprehend the whole system function as it has tightly coupled parts. The function of one is dependent on the other. Thus the ADS provide on-line expansion facility to address this problem.

\section{System Modification}

Companies invest heavily in the installation and commissioning of manufacturing systems. When a need arises to modify part of the system, the whole system has to be shut down resulting in the loss of valuable time. Therefore a system capable of on-line expansion and on-line maintenance/test would be highly desirable ${ }^{6,7}$.

It is therefore imperative that the system modifications and additions are carried out on-line, during its operation. In addition, when any new part of the system is added to the main system, it becomes necessary to carry out its functional

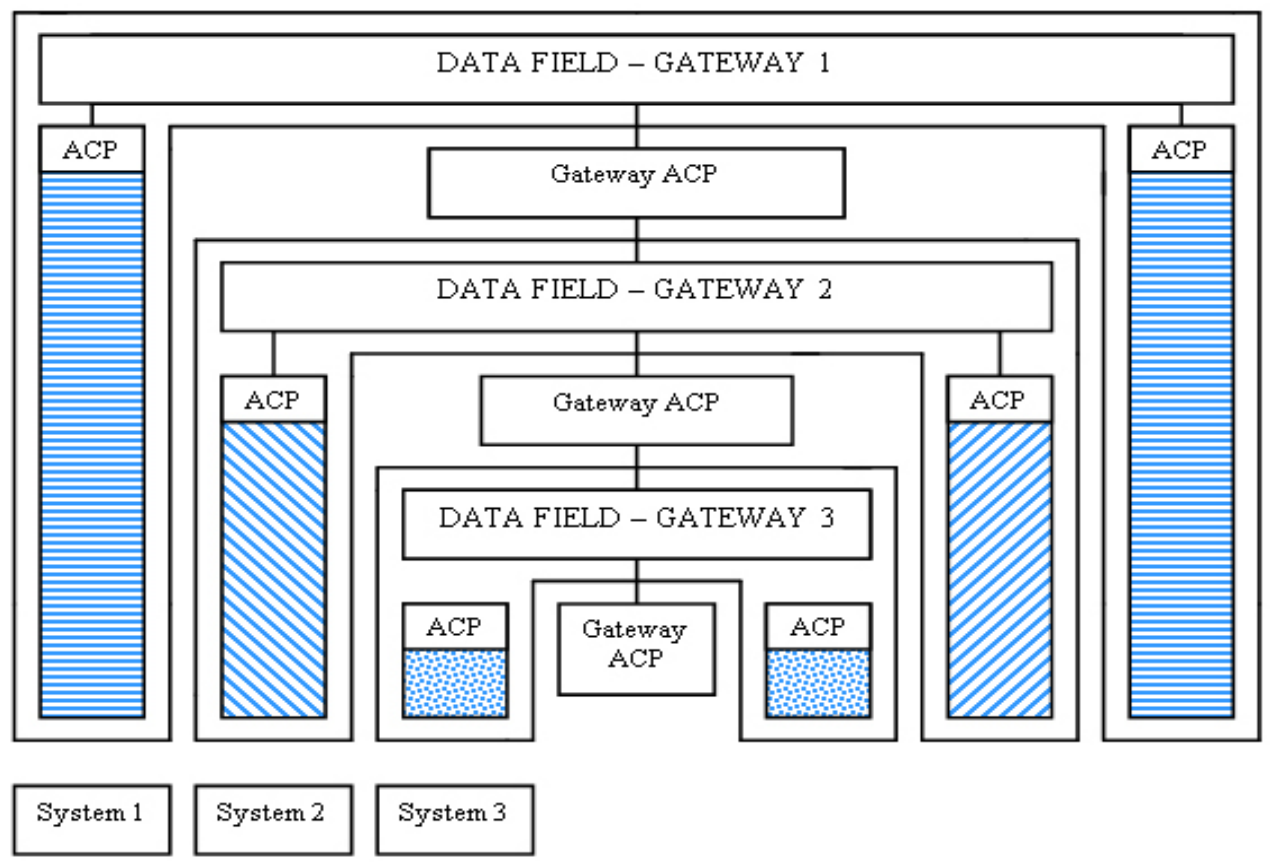

Fig. 3: The gateway mechanism connecting various application systems in a heterogeneous system 
checks to ascertain as to whether the new subsystem can carry out its designed task. Thus on-line testing and on-line maintenance of these manufacturing systems should be possible.

\section{Customer driven Manufacturing}

At the time businesses are required to monitor customer trends/demands and then quickly adjust the production process to meet these demands in today's competitive environment. Conventional manufacturing involves set patterns of mass produced items managed by a central computing facility. Both the information and control subsystems perform independently. The information system manages the production scheduling, pricing, sales and accounts while the control system regulates the control of machines. The production schedule is better comprehended by workers on the factory floor and they are in a better position to modify the schedule according to the state of machines serviceability coupled with information regarding customer's trend/demands. Thus the integration of information and control is fruitful to boost productivity.

\section{Flexibility Function}

A number of machines work in unison to manufacture a product. In order to regulate the working of these machines and the operators, appropriate scheduling needs to be carried out. Due to changes in demand and essential maintenance and repair to be carried out on these machines, the scheduling function needs to be flexible to cater for these requirements.

\section{A Typical ADS}

The ADS concept has been successfully applied to a vast number of systems including highly reliable steel manufacturing systems ${ }^{8}$. A steel production system will be briefly described in this section. The requirements of steel production are in line with the provisions of the ADS. The successful implementation of ADS in steel production is due to two main reasons. In the first case a feedback control system is used to monitor the data from sensors and management modules of the plant. The actuators, monitors and sensors though incorporate artificial intelligence, these are still device dependent. Their functions can be capsulated which correspond to the ADS subsystems ${ }^{8}$. Secondly, these capsules are inter-connected via the data itself, not according to the process situation. The capsule data can be uniformly defined both for the input and output.

In the ADS, the subsystems initiate their work after getting complete data from the ADF. These capsules are thus ideally suited for ADS application. The interaction among three functional layers of a steel production unit is depicted in Fig. 4.

The on-line maintenance and test provision in the aforementioned steel production plant boosted the productivity by $30 \%^{8}$. However, extensive manpower training is required to be carried out in a system employing the ADS technology. Also actuators and switching devices are tending to be incorporating artificial intelligence. This allows these devices to be capsulated and therefore could be connected in an ultra distributed system involving thousands of such devices ${ }^{8}$.

\section{Current State and Future Trends}

ADS have progressed to data intensive system applications since their inception. The major thrust of research effort has been to integrate the information and control functions in the conventional manufacturing systems ${ }^{9}$. The ADS in the integrated form realizes the goal of on-line development and maintenance ${ }^{10}$. There is a dire need to provide a software architecture that is capable of controlling correct synchronization, reconfiguration and reliability ${ }^{11,13}$. Functional reliability of manufacturing systems employing ADS is equally important. Autonomous successive construction technology without stopping system operation for a real time system has recently been reported ${ }^{14}$. The authors demonstrate that the optimal method for system construction is through small subsystems. The functional reliability and on-line functional availability have been characterized to be the best index for system assurance indication.

Recently the ADS has been given a new impetus by the unpredictable market forces as new endeavours are made in the field of information technology, i.e, LSI and optical fibre in addition to heterogeneous market requirements ${ }^{15}$. Thus the major advancement in ADS technology has been LAN-centric manufacturing. However, in the near 


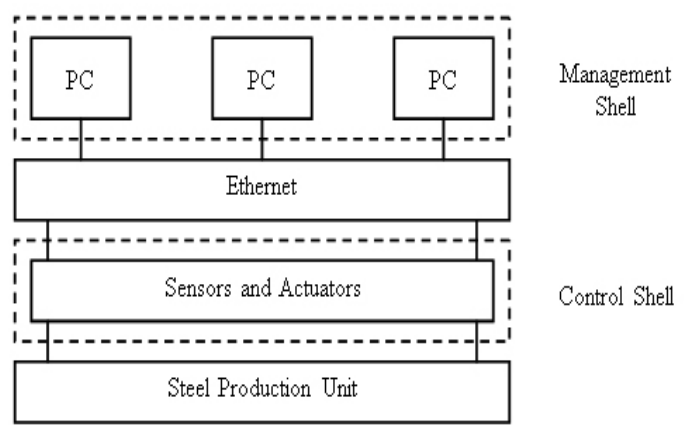

Fig. 4: A symbolic representation of a steel production unit

future, the overall cost of ADS based manufacturing systems will be reduced by up to $80 \%$ of its IT component as both the computing resources and the underlying communication will be organized as an infrastructure community ${ }^{15}$. This community will be managed as autonomous community components thus relieving the human resource from managing the computing systems. Therefore autonomous community technology is one of the latest paradigms of ADS.

\section{CONCLUSION}

The ADS concept has been reviewed in this paper in addition to its application to a steel manufacturing system. Distributed systems that include many autonomous entities can benefit from the ADS technology. The ADS is an integrated set of subsystems that can be modified and tested online in addition to being fault tolerant. Conventional Manufacturing trends have been identified to be giving way to dynamic volume production systems in response to user trends and demands. The future of ADS is seen to be associated with autonomous community concept where each component of the community will be autonomously handled. The ADS have been instrumental in boosting productivity and lowering costs in manufacturing systems and have been approved as the de-facto standard in factory automation..

\section{REFERENCES}

1. Duffie, N.A., R. Chitturi, J. Mou," Fault-tolerant Hierarchical Control of Heterogeneous Manufacturing System Entities," Journal of Manufacturing Systems, 7(4): 315-328, 1988.

2. Jones, A.T and C.R Mclean," A Proposed Hierarchical Control Model for Automated Manufacturing Systems," Journal of Manufacturing Systems, 5(1):15-25, 1986.

3. DeviceNet Specifications," Open DeviceNet Vendor Association, Inc., April 22, 1996.

4. Mori, K. et al," Proposition of Autonomous Decentralization Concept," Journal of IEEE Japan, 104(12): 303-310, 1984.

5. Kinji Mori," Expandable and Fault-Tolerant Computers and Communication SystemsAutonomous Decentralized Systems," 4 th International Symposium on Computers and Communications, ISCC'99, 228-234, 1999.

6. M. Oku, M. Omura, M. Perrone and M Roth," $\mathrm{Hi}$-Cell Architecture and the Project Model for Manufacturing Autonomous Decentralized Systems," Proceedings of International Symposium on ADS, ISADS'93, 398-405,
1993.

7. M.Omura M.Oku," Hi-Cell Architecture for Manufacturing systems," Proceedings of International Symposium on ADS, ISADS'95, 154-161, 1995.

8. Hiroshi Kuwahara," Experiences Teach us the Future of Autonomous Decentralized Systems," Proceedings of International Symposium on ADS, ISADS'97, 169-175, 1997.

9. Shigeloshi Smeshiima, Kawano, K., Kumayama, J., Ito, T.," An ADS Architecture and Techniques for On-Line Development and Maintenance," Proceedings of International Symposium on ADS, ISADS'97, 121-128, 1997.

10. Hiroshi Wataya., Hayashi, K., Toyouchi, J " Integration of Control and Information Systems by Open ADS Architecture and its Applications for Distributed Manufacturing Systems," Proceedings of International Symposium on ADS, ISADS'97, 147-154, 1997.

11. Liskov, B., D Curtis, P. Johnson, R. Scheifler," 
Implementation of Argus," Proc. 11 $1^{\text {th }}$ Symposium on Operating Systems, 111122, 1987.

12. Magee, J. J. Kramer, M Sloman," Constructing Distributed Systems in Comic," IEEE Trans. On Software Engineering, 15(6): 663-675, 1989.

13. Alvin S. Lim," A Uniform Software Architecture for Cooperation, Reliability and Reconfiguration of ADS," Proceedings of
International Symposium on ADS, ISADS'95, 33-39, 1995.

14. Kazou Kera, keisnke Bekki, Hideki Osumi, Kinji Mori," Autonomous Successive Construction Technology Without Stopping System Operation for Real-Time Systems," Proceedings of International Symposium on ADS, ISADS'03, 266-273, 2003.

15. Kinji Mori," Autonomy and Community," Proceedings of International Symposium on ADS, ISADS' 03, 266-273, 2003. 